\title{
Primary glioblastoma of the cerebellar vermis: A case report
}

\author{
XIAOLIN JING ${ }^{*}$, GUOHUA SHEN* ${ }^{*}$, MINGGANG SU, RONG TIAN and ZHIYUN JIA \\ Department of Nuclear Medicine, West China Hospital, Sichuan University, Chengdu, Sichuan 610041, P.R. China
}

Received August 8, 2014; Accepted April 21, 2015

DOI: $10.3892 / 01.2015 .3188$

\begin{abstract}
Cerebellar glioblastoma is a rare adult tumor. The accurate diagnosis of cerebellar glioblastoma is important for establishing a suitable therapeutic schedule. However, it is occasionally difficult to diagnosis these tumors. Clinical presentation, computed tomography (CT) and magnetic resonance imaging can provide useful information, but they may not lead to a definitive diagnosis. Positron emission tomography/computed tomography (PET/CT) may provide a novel way of forming a differential diagnoses. The lesions of glioblastoma multiforme (GBM) rarely occur in the cerebellum, with prior studies reporting that only $0.4-3.4 \%$ of all GBM tumors occur here. In the current study, a case of primary cerebellar glioblastoma is presented and the physiopathology, clinical presentation, diagnosis, differential diagnosis, treatment and general outcome of this disease are discussed. A 61-year-old female presented with nausea, vomiting, balance problems and cerebellar signs. Cranial magnetic resonance imaging (MRI) and positron emission tomography/computed tomography (PET/CT) examination demonstrated one regular contour of a mass lesion in the cerebellar vermis. Following surgery, glioblastoma was histologically confirmed. The outcome of the patient was favorable after 18 months of follow-up. Cerebellar GBM should be considered in the differential diagnosis of a cerebellar mass lesion, and PET/CT may provide a novel identification method for different cerebellar mass lesions.
\end{abstract}

\section{Introduction}

Glioblastoma multiforme (GBM) is the most frequently occurring malignant primary brain tumor in adults. These tumors generally develop in the fifth and sixth decades of life $(1,2)$.

Correspondence to: Dr Zhiyun Jia, Department of Nuclear Medicine, West China Hospital, Sichuan University, 37 Guo Xue Xiang, Chengdu, Sichuan 610041, P.R. China

E-mail: zhiyunjia@hotmail.com

${ }^{*}$ Contributed equally

Key words: cerebellar, glioblastoma, fluorodeoxyglucose, positron emission tomography/computed tomography, magnetic resonance imaging
However, these lesions rarely occur in the cerebellum, with prior studies reporting that only $0.4-3.4 \%$ of all GBM tumors occur in this location (3-5). Hypertension, impaired balance and gait disturbance are typical clinical manifestations (1). The best treatment for cerebellar glioblastoma is removal of as much of the tumors as possible but keeping surgical morbidity to a minimum (2). The prognosis of cerebellar glioblastoma is similar to that of anaplasic astrocytoma (1). In the current study, a case of primary cerebellar glioblastoma is presented, and the physiopathology, clinical presentation, diagnosis, differential diagnosis, treatment and general outcome of this disease is discussed.

\section{Case report}

A 61-year-old female with no other medical history was referred to West China Hospital of Sichuan University (Chengdu, China) and presented with nausea, vomiting and balance problems that had persisted for $>1$ month. A neurological examination demonstrated cerebellar signs, including positive bilateral finger-nose and knee-shin tests. Tumor marker analysis and other blood tests were negative. Cranial magnetic resonance imaging (MRI) showed one regular contour of a mass lesion in the cerebellar vermis. The mass was present as a hypointense and hyperintense lesion in the cerebellar vermis in T1- and T2-weighted images, respectively (Fig. 1A and B). The mass had well-defined borders and large areas of central necrosis. T1-weighted magnetic enhanced imaging showed a well-defined, heterogeneously ring-enhancing lesion, with one additional enhanced node (Fig. 1C and D). The provisional diagnosis was of a metastatic lesion of the cerebellum. The patient signed the written informed consent and then underwent a positron emission tomography/computed tomography (PET/CT) examination. There was no abnormal glucose uptake on coronal PET imaging (Fig. 2A). Cerebral CT showed one hypodense lesion with a nodule alongside the cerebellar vermis (Fig. 2B). In PET imaging, the nodule was observed to have much higher glucose uptake than other tissues (Fig. 2C and D). Therefore, the patient was diagnosed with primary brain tumors.

The patient underwent a cerebellar lesion resection at West China Hospital of Sichuan University (Chengdu, China) and the pathology of the lesion diagnosed a glioblastoma (class IV; Fig. 3). Following the surgery, histopathological examination revealed a cellular tumor, which was consistent with glioblastoma that met the World Health Organization criteria of grade 4 astrocytoma. The appearance of the tumor was consistent 

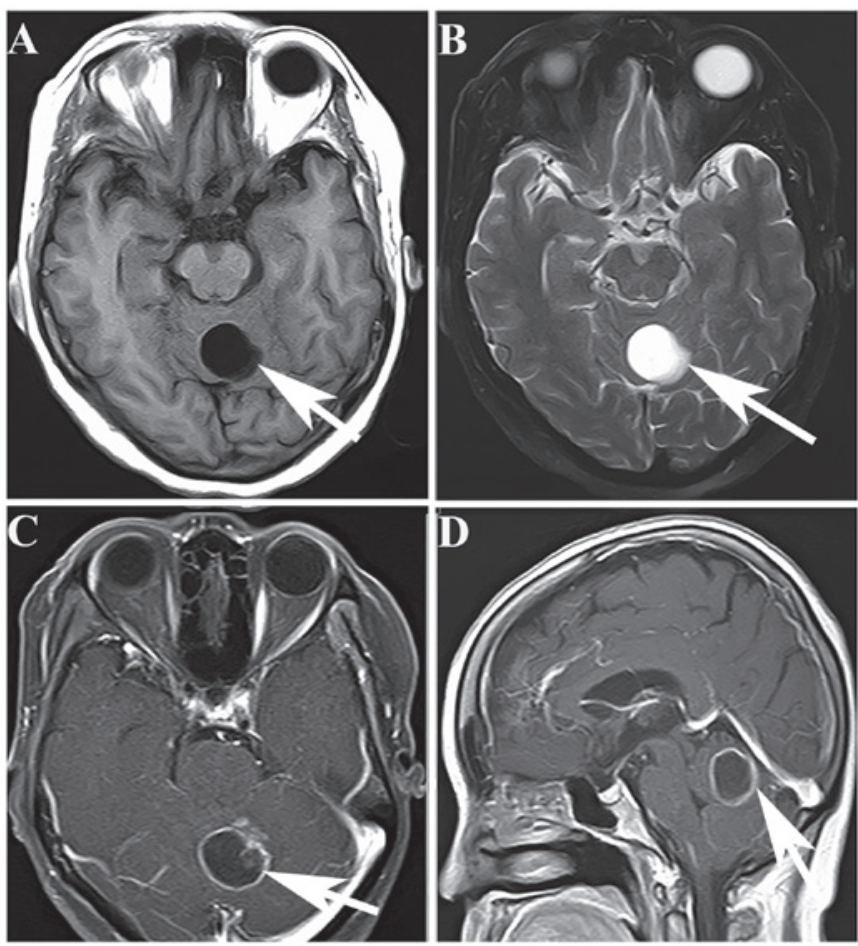

Figure 1. Cranial magnetic resonance imaging showing one lesion in the cerebellar vermis. (A) T1-weighted image showing a well-defined hypointense tumor in the vermis of the cerebellum (arrow). (B) T2-weighted image showing a well-distributed hyperintense lesion without evident surrounding edema (arrow). (C and D) T1-weighted magnetic resonance enhanced images showing a well-defined, ring-enhancing lesion with one additional enhanced nodule (arrow)

with necrosis, pseudo-palisading and vascular hyperplasia. Photomicrograph of tumor sections demonstrated an intense cellular density and nuclear pleomorphism, which was characterized by marked anisokaryosis and larger nuclei than is normal. The cells had an abundant and eosinophilic cytoplasm and umerous mitoses were observed (Fig. 3).

The patient had modest brain edema following the surgery for a few days, but improved immediately after diuretics were administered. The neurologic signs improved and the clinical presentation disappeared. The patient did not receive radiotherapy or chemotherapy after the surgery. There was no sign of tumor recurrence after 18 months.

\section{Discussion}

Cerebellar GBM in adults is rare, and thus comprises only a small proportion of all GBM tumors of the brain. GBM of the cerebellum can be observed in all age groups. A study has shown that the male-to-female ratio for this disease is $2: 1$, and that $270 \%$ of the tumors occur in adults (average age, 46.7 years), while $30 \%$ occur in children (average age, 10.4 years) (6). GBM is a stage 4 tumor according to the World Health Organization classification (7). The prognosis of this entity is uncertain, and factors associated with prognosis have been unclear. According to the case study of a 41-year-old male with a cerebellar glioblastoma developing in the cerebellar hemisphere who was treated 35 years previously by radiotherapy, radiation may be a cause for inducing GBM (8): The patient had medulloblastoma (MB) in the

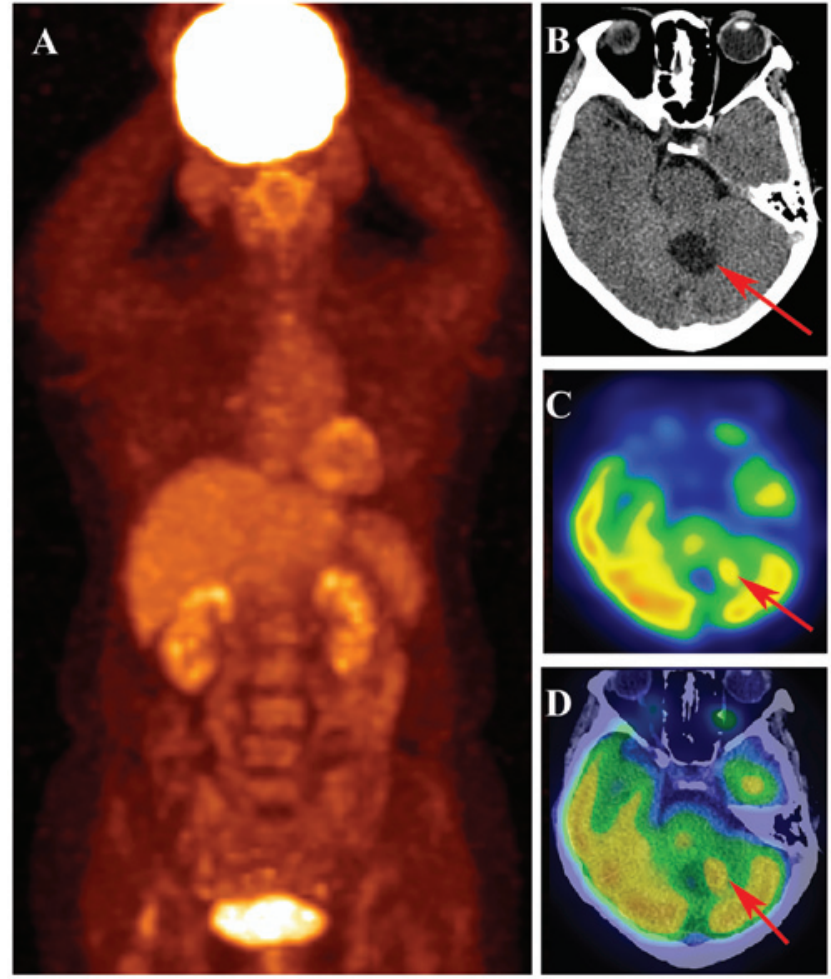

Figure 2. (A) Coronal positron emission tomography (PET) image showing no abnormal glucose uptake. (B) Computed tomography scan showing a round cystic mass with more hypodense expression than normal brain tissue. In addition, there is a hyperdense nodule alongside the mass (arrow). (C and D) In the PET and PET/CT images, the nodule (arrow) shows much higher glucose uptake compared with the surrounding brain tissue.
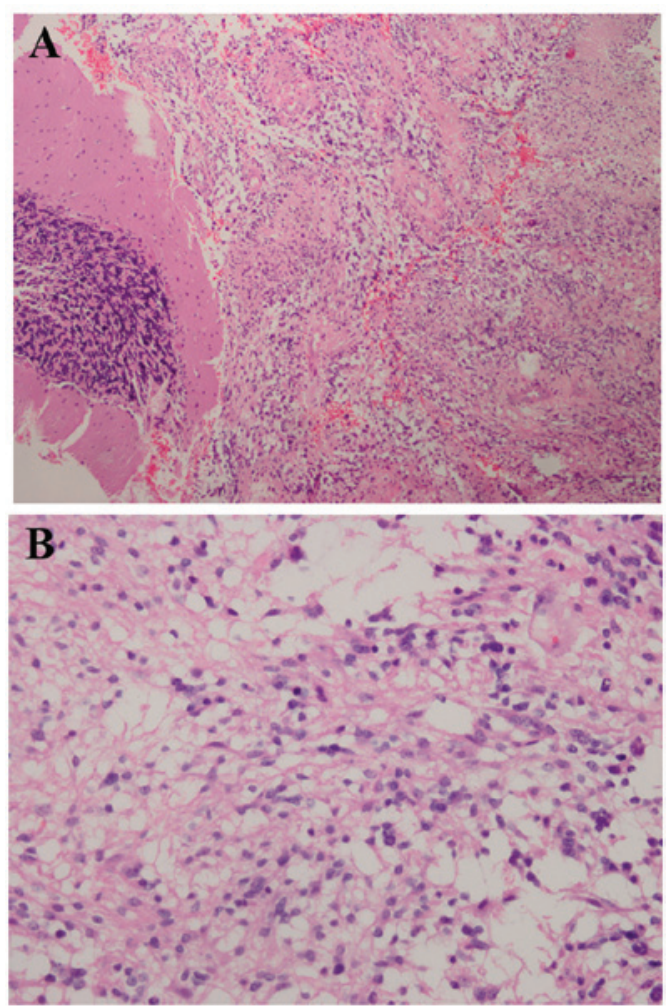

Figure 3. Tumor sections showing an intense cellular density and nuclear pleomorphism. (A) The tumor cells are characterized by marked anisokaryosis; the nuclei are larger than normal. The cells have an abundant and eosinophilic cytoplasm. Numerous mitoses are observed. Hematoxylin and eosin stain; (A) x100 and (B) x400 magnification. 
cerebellar vermis, and postoperatively he received a total of 40 Gy radiations to the whole brain and 30.5 Gy to the spine without chemotherapy (8).

Patients with GBM typically present with increased hypertension, impaired balance and gait disturbance (1). Upon examination, patients exhibit cerebellar signs, as occurred in the present case. The symptoms of dizziness, mental confusion and neck pain can also be present (9). A diagnosis of cerebellar GBM is not usually pre-operatively suspected, although certain CT and MRI characteristics may indicate it $(6,9)$. Cerebellar metastases, hemangioblastomas, brain abscesses and anaplastic astrocytomas are common differential diagnoses in adults. Brain abscesses often have symptoms of infection. Abscess walls are mostly smooth without nodules. Enhanced CT and MRI scans show incomplete ring-like reinforcements, which are thin and uniform. Hemangioblastomas always occur singly, and $>90 \%$ occur in the cerebellar hemisphere. The CT and MRI indications are similar to those in the present case. The pathology of a lesion can only be confirmed after surgery. Brain metastases always have necrosis within them and show greater than one lesion with marked peripheral edema $(1,2,4,9)$. The history of a primary tumor is important. In fact, to identify these diseases, a number of different examinations are required, and they may take a long time to complete. At the West China Hospital of Sichuan University, patients are provided with PET/CT examinations according to whether lesions take up glucose or not, so that this disease can be identified. However, reports of signs of different brain tumors in PET/CT scans are rare. As PET/CT scans become increasingly common, they may become a novel method for identifying brain tumors.

Cerebellar glioblastoma treatment is usually palliative, consisting of surgery, radiotherapy and chemotherapy $(1,2)$. Decisions regarding surgery depend on factors such as patient age, performance, diseased region in the brain and resectability of the tumor $(1,2,4,6)$. Subsequent to surgery, patients can be treated with radiotherapy and chemotherapy. However, GBM is the highest graded astrocytoma and the recurrence rate following surgery is high. Moreover, the prognosis of patients with glioblastoma is poor, with a 2-year survival rate of $10 \%$ and $<5 \%$ of the patients surviving for a long period of time. The mean survival time for patients with cerebellar glioblastoma after the beginning of symptomatology has been reported to be $12-19$ months $(6,10)$.

The present study reports a rare case of cerebellar GBM. PET/CT examination may provide a novel method for diagnosing GBM and providing differential diagnoses for metastases, abscesses and hemangioblastomas in the cerebellum.

\section{Acknowledgements}

This study was supported by the National Natural Science Foundation (grant nos. 81271532 and 30900378) and the Fundamental Research Funds for the Central Universities (project no. 2015SCU04B09).

\section{References}

1. El El Maaqili MR, Hossini A, El Fatemi N, et al: Primary glioblastoma of the cerebellum in a 19-year-old woman: a case report. J Med Case Rep 6: 329, 2012.

2. Grahovac G, Tomac D, Lambasa S, et al: Cerebellar glioblastomas: Pathophysiology, clinical presentation and management. Acta Neurochir 151: 653-657, 2009.

3. Babu R, Sharma R, Karikari IO, et al: Outcome and prognostic factors in adult cerebellar glioblastoma. J Clin Neurosci 20: 1117-1121, 2013.

4. Kuroiwa T, Numaguchi Y, Rothman MI, et al: Posterior fossa glioblastoma multiforme: MR findings. AJNR Am J Neuroradiol 16: 583-589, 1995.

5. Roth JG and Elvidge AR: Glioblastoma multiforme: A clinical survey. J Neurosurg 17: 736-750, 1960.

6. Mattos JP, Marenco HA, Campos JM, et al: Cerebellar glioblastoma multiforme in an adult. Arq Neuropsiquiatr 64: 132-135, 2006.

7. Louis DN, Ohgaki H, Wiestler OD, et al: The 2007 WHO classification of tumours of the central nervous system. Acta Neuropathol 114: 97-109, 2007.

8. Hamasaki K, Nakamura H, Ueda Y, et al: Radiation-induced glioblastoma occurring 35 years after radiation therapy for medulloblastoma: case report. Brain Tumor Pathol 27: 39-43, 2010.

9. Demir MK, Hakan T, Akinci O and Berkman Z: Primary cerebellar glioblastoma multiforme. Diagn Interv Radiol 11: 83-86. 2005.

10. Kulkarni AV, Becker LE, Jay V, et al: Primary cerebellar glioblastomas multiforme in children. Report of four cases. J Neurosurg 90: 546-550, 1999. 